\title{
A faunistic study on the leafhoppers of northwestern Iran (Hemiptera, Cicadellidae)
}

\author{
Tandis Abdollahi', Ali Reza Jalalizand', Fariba Mozaffarian², Michael Wilson³ \\ I Department of Plant protection, Faculty of Agriculture, Islamic Azad University, Isfahan (Khorasgan) Branch, \\ Isfahan, Iran 2 Insect Taxonomy Research Department, Iranian Research Institute of Plant Protection, Tehran, \\ 19395, P.O. Box 1454, Iran 3 Department of Natural Sciences, National Museum of Wales, Cardiff, U.K.
}

Corresponding author: Tandis Abdollahi (tandis_ab@yahoo.com)

Academic editor: Mick Webb | Received 3 December 2014 | Accepted 11 March 2015 | Published 16 April 2015

http://zoobank.org/70F28058-13AA-4220-A076-FDC6C46BC87A

Citation: Abdollahi T, Jalalizand AR, Mozaffarian F, Wilson M (2015) A faunistic study on the leafhoppers of northwestern Iran (Hemiptera, Cicadellidae). ZooKeys 496: 27-51. doi: 10.3897/zookeys.496.9059

\begin{abstract}
The leafhopper fauna of northwestern Iran: Azarbaijan-e-Sharghi, Azarbaijan-e-Gharbi and Ardabil provinces is listed from previously published records and from our current work. Sixty-nine species are included with four species (Mogangella straminea Dlabola, 1957, Doratura stylata (Boheman, 1847), Macrosteles sordidipennis (Stål, 1858) and Psammotettix seriphidii Emeljanov, 1962) listed as new for Iran and Balclutha punctata (Fabricius, 1775), as a new record for the region. A distribution map of the species in northwestern Iran is given.
\end{abstract}

\section{Keywords}

Cicadellidae, leafhoppers, fauna, Azarbaijan-e-Sharghi, Azarbaijan-e-Gharbi, Ardabil

\section{Introduction}

The Auchenorrhyncha consists of approximately 42000 described worldwide species which have adopted varied life habits (Larivière et al. 2010). Moreover, they play an important role in the food chains due to their high biomass in the herb layer and provide a food source for other insects and animals (Nickel 2003). 
The family Cicadellidae (leafhoppers), is the largest family within the Hemiptera, with approximately 19,500 described species in more than 40 subfamilies (Oman et al. 1990). Metcalf (1962-1968) considered the leafhoppers to represent a superfamily (the Cicadelloidea) and divided them into a number of families, currently subfamilies or tribes. Following Dietrich (2005), Cicadellidae are included in the superfamily Membracoidea with the Membracidae (treehoppers). Most Cicadellidae species tend to feed from phloem fluid (except some Cicadellinae and most Typhlocybinae) (Biedermann and Niedringhaus 2009). Moreover, some species may cause both direct and indirect damage during their feeding activity, which is sometimes economically important. The most important form of indirect damage is caused by phytoplasmas and viruses, vectored mostly by Cicadellidae (Weintraub and Beanland 2006).

The earliest available record of Auchenorrhyncha from Iran is Gardenhire (1959) who recorded some species as agricultural pests. Jiri Dlabola, from the Czech Republic, studied considerable numbers of Auchenorrhyncha species from Iran in the 1970s, which led to the discovery of more than 100 new Cicadellidae species in a long series of papers (Dlabola 1974, 1977, 1980, 1981, 1982, 1983, 1985). More recently other authors have published on the fauna: Karimzadeh et al. (1998); Haghshenas and Khajeali (2000); Lashkari et al. (2009); Taghizadeh et al. (2010); Mozaffarian and Taghizadeh (2010); Mozaffarian and Emeljanov (2010); Mozaffarian et al. (2010); Mozaffarian and Sanborn (2010, 2012, 2013); Mozaffarian and Gnezdilov (2011); Gnezdilov and Mozaffarian (2011); Moazaffarian and Wilson (2011); Moosavi and Sadeghi Namaghi (2012); Mozaffarian (2012a, b); Taghizadeh (2012); Zohdi et al. (2012); AghagoliMarzijarani et al. (2013), Mozaffarian (2013) and Abdollahi et al. (2013, 2014). There have been a wide range of researchers who mainly focused on the Auchenorrhyncha as pests in both agricultural and forest ecosystems in Iran such as: Gharib (1966); Kheyri and Alimoradi (1969); Kheyri (1989, 1992); Rajabi and Mirzayans (1989); Behdad (1992, 1993); Khajehali et al. (2001); Nematollahi and Khajehali (2000); Yarmand et al. (2006); Aghagoli-Marzjarani et al. (2010); Taghizadeh et al. (2010) amongst others.

Northwestern Iran (the study area) covers nearly 100,503 square kilometers and consists of three provinces: Azarbaijan-e-Sharghi, Azarbaijan-e-Gharbi and Ardabil. It is located in Irano-Turanian zoogeographical region (Firouz 2005) and in the northwest plateau of Iran. It is limited between the Caspian Sea and Caspian district in the east, Caucasus mountains in the north, Anatolian Plateau and Mesopotamian region in the west and a part of Zagros, called Humid Zagros, in the south. Hence, it is expected that the fauna of this area will be influenced by the faunal elements of all mentioned regions rather than just the Iranian Plateau. The area is considered to be the crossroads of the two main mountains of Iran (Alborz and Zagros), a part of Alpine Himalayan orogenic belt (Dewey et al. 1986) with deep valleys and has a variety of altitudes from $256 \mathrm{~m}$ to $2896 \mathrm{~m}$. It is differentiated from other parts of Iran by the highest latitude $\left(39^{\circ} 40^{\prime} \mathrm{N}\right)$ and the coldest recorded temperature $\left(-35^{\circ} \mathrm{C}\right)($ Hedge and Wendelbo 1978). Zarudny (1911) considered this part of Iran as a zoogeographic zone, with a fauna similar to the Caucasus. This area was also considered as a different 


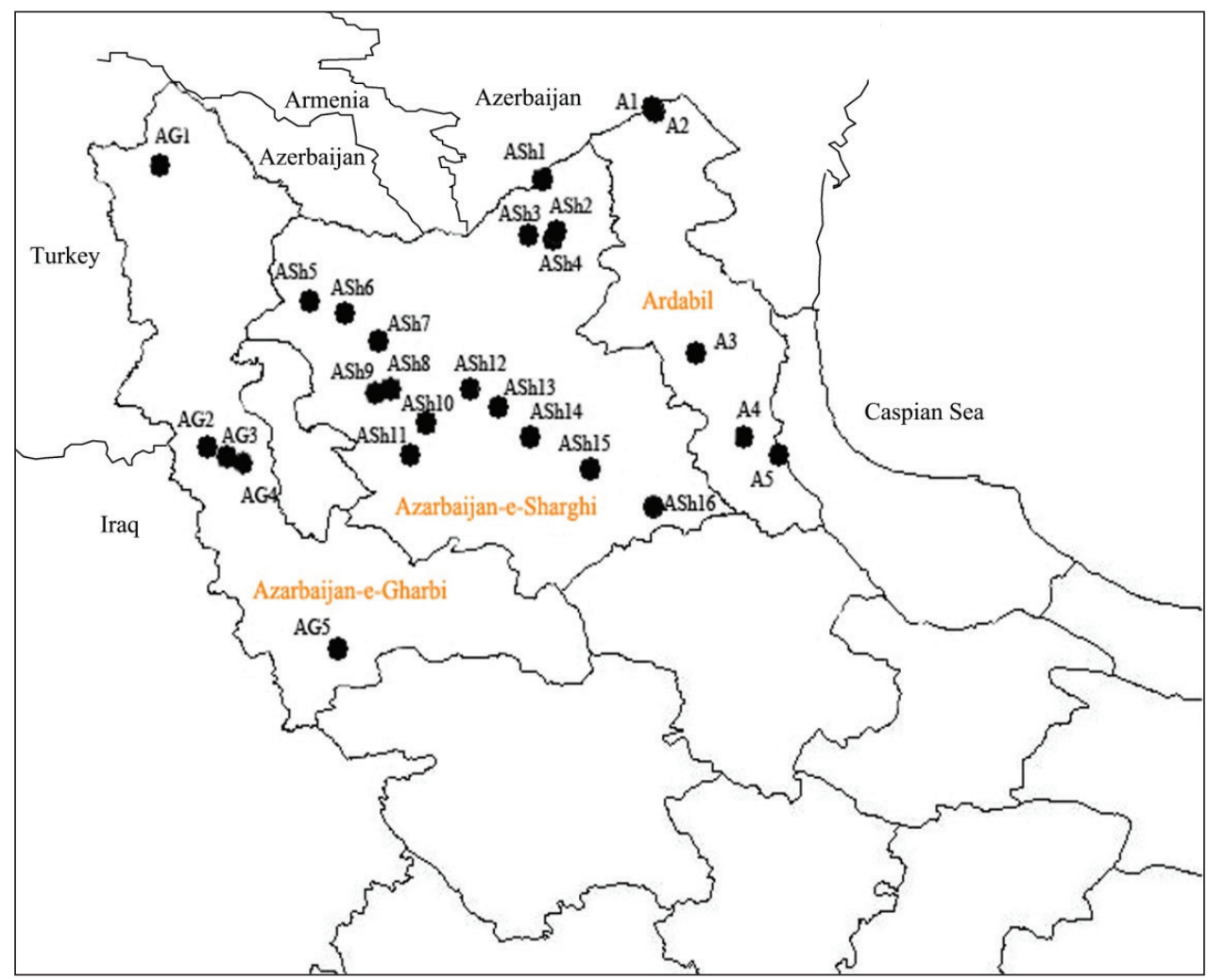

Figure I. Distribution map of leafhoppers in northwestern Iran (For codes see Table 1).

area from other parts of Zagros by Emeljanov (1974). Hedge and Wendelbo (1978) recognized part of Iran as an endemic zone named Armeno-Kurdic due to the distribution patterns of endemic phanerogamic plants.

The aim of this research was to collect and identify the leafhoppers in northwestern Iran and to prepare a checklist as a starting point for gathering the sporadic publications and studying the fauna for the whole of Iran. A total number of 69 species belonging to 11 subfamilies are recorded.

\section{Material and methods}

The present study was carried out in three northwestern provinces including, Azarbaijan-e-Sharghi, Azarbaijan-e-Gharbi and Ardabil provinces (Figure 1). During 2007 (August, September and January) and 2008 (January) field trips were made and leafhopper specimens were collected using a sweep net. A total of 2340 specimens consists of newly collected specimens along with other specimens located in the Hayk Mirzayans Insect Museum (Tehran, Iran, which had been collected since 1968) were studied and identi- 
Table I. List of the localities, their coordinates and the codes

\begin{tabular}{c|c|c|c}
\hline Locality names & Coordinates & Locality names & Coordinates \\
\hline Ajabshir & $37^{\circ} 35^{\prime} \mathrm{N}, 46^{\circ} 11^{\prime} \mathrm{E}$ (ASh11) & Moghan & $39^{\circ} 37^{\prime} \mathrm{N}, 47^{\circ} 47^{\prime} \mathrm{E}$ (A1) \\
\hline Bonab & $37^{\circ} 26^{\prime} \mathrm{N}, 45^{\circ} 57^{\prime} \mathrm{E}$ (ASh9) & Eskanlu & $39^{\circ} 12^{\prime} \mathrm{N}, 47^{\circ} 04^{\prime} \mathrm{E}$ (ASh1) \\
\hline Bostanabad-Siah chaman & $37^{\circ} 41^{\prime} \mathrm{N}, 46^{\circ} 59^{\prime} \mathrm{E}$ (ASh14) & Parsabad & $39^{\circ} 36^{\prime} \mathrm{N}, 47^{\circ} 49^{\prime} \mathrm{E}$ (A2) \\
\hline Heiran & $37^{\circ} 41^{\prime} \mathrm{N}, 48^{\circ} 24^{\prime} \mathrm{E}$ (A4) & Sarein & $38^{\circ} 11^{\prime} \mathrm{N}, 48^{\circ} 05^{\prime} \mathrm{E}$ (A3) \\
\hline Kaleibar, Arabshahi & $38^{\circ} 51^{\prime} \mathrm{N}, 47^{\circ} 08^{\prime} \mathrm{E}$ (ASh4) & Siah chaman-Basmenj & $37^{\circ} 52^{\prime} \mathrm{N}, 46^{\circ} 46^{\prime} \mathrm{E}$ (ASh13) \\
\hline Kaleibar, $1863 \mathrm{~m}$ & $38^{\circ} 52^{\prime} \mathrm{N}, 46^{\circ} 58^{\prime} \mathrm{E}$ (ASh3) & Sufian & $38^{\circ} 16^{\prime} \mathrm{N}, 45^{\circ} 58^{\prime} \mathrm{E}$ (ASh7) \\
\hline Kaleibar, 1732m & $38^{\circ} 54^{\prime} \mathrm{N}, 47^{\circ} 09^{\prime} \mathrm{E}$ (ASh2) & Tabriz & $37^{\circ} 58^{\prime} \mathrm{N}, 46^{\circ} 03^{\prime} \mathrm{E}$ (ASh8) \\
\hline Kandovan & $37^{\circ} 46^{\prime} \mathrm{N}, 46^{\circ} 17^{\prime} \mathrm{E}$ (ASh10) & Tabriz-Shabestar & $38^{\circ} 15^{\prime} \mathrm{N}, 45^{\circ} 58^{\prime} \mathrm{E}$ (ASh7) \\
\hline Khalkhal & $37^{\circ} 35^{\prime} \mathrm{N}, 48^{\circ} 38^{\prime} \mathrm{E}$ (A5) & Tabriz-Bostanabad & $37^{\circ} 58^{\prime} \mathrm{N}, 46^{\circ} 35^{\prime} \mathrm{E}$ (ASh12) \\
\hline Mahabad & $36^{\circ} 27^{\prime} \mathrm{N}, 45^{\circ} 42^{\prime} \mathrm{E}$ (AG5) & Uromieh & $37^{\circ} 25^{\prime} \mathrm{N}, 47^{\circ} 42^{\prime} \mathrm{E}$ (AG3) \\
\hline Maku & $39^{\circ} 17^{\prime} \mathrm{N}, 44^{\circ} 31^{\prime} \mathrm{E}$ (AG1) & Uromieh, Mirzabad & $37^{\circ} 32^{\prime} \mathrm{N}, 45^{\circ} 04^{\prime} \mathrm{E}$ (AG4) \\
\hline Marand & $38^{\circ} 25^{\prime} \mathrm{N}, 45^{\circ} 45^{\prime} \mathrm{E}$ (ASh6) & Uromieh-Sarv & $37^{\circ} 38^{\prime} \mathrm{N}, 44^{\circ} 50^{\prime} \mathrm{E}$ (AG2) \\
\hline Miyaneh-Siah chaman & $37^{\circ} 30^{\prime} \mathrm{N}, 47^{\circ} 23^{\prime} \mathrm{E}$ (ASh15) & Zonuschay & $38^{\circ} 29^{\prime} \mathrm{N}, 45^{\circ} 31^{\prime} \mathrm{E}$ (ASh5) \\
\hline Miyaneh-Zanjan & $37^{\circ} 17^{\prime} \mathrm{N}, 47^{\circ} 48^{\prime} \mathrm{E}$ (ASh16) & & \\
\hline
\end{tabular}

fied. The identifications were made using the works of Le Quesne (1965, 1969), Ribaut (1952), Biedermann and Niedringhaus (2009) and Emeljanov (1997). Vouchers of all species are deposited in the Hayk Mirzayans Insect Museum. In addition to the identification, literature records were also taken into consideration and a distribution map for the leafhoppers of northwestern Iran was prepared by ARCMAP version 9.3.0.1770.

\section{List of taxa}

The genera and species from northwestern Iran recorded through the present study and other publications are as follows (* indicates species not found in the present study). For those species with specimens examined, a reference to the authority used for the identification is included in parenthesis following the taxon heading. The classification used follows mainly Oman et al. (1990) with changes based on more recent literature e.g., Zahniser and Dietrich (2013) for Deltocephalinae.

\section{Subfamily: Agalliinae \\ Tribe: Agalliini}

\section{Agallia firdausica Dlabola, 1981*}

Localities. Sufian (Dlabola 1981) (Fig. 1, ASh7).

Worldwide distribution. Iran; Saudi Arabia (Dlabola 1980). 


\section{Anaceratagallia laevis (Ribaut, 1935)}

Anaceratagallia laevis: Le Quesne 1965: 52, figs 267-268.

Material examined. Azarbaijan-e-Sharghi: $1 \hat{\jmath}, 2$, Ajabshir, Yaichi village, $1922 \mathrm{~m}$, 37³5'27.2"N, 46¹1'03.7"E, 15.January.2008, leg. Mozaffarian (Fig. 1, ASh11).

Dlabola (1981) reported this species from Sufian (Fig. 1, ASh7).

Worldwide distribution. East Palaearctic, Europe (Albania, Britain I., Bulgaria, Canary Is., Channel Is., Cyprus, French mainland, Greek mainland, Hungary, Italian mainland, Moldova, Portuguese mainland, Romania, South Russia, Sardinia, Sicily, Spanish mainland, Ukraine, Yugoslavia), Near East, North Africa (De Jong 2013).

\section{Austroagallia sinuata (Mulsant \& Rey, 1835)}

Austroagallia sinuata: Le Quesne 1965: 50, figs 253-255, 257.

Material examined. Azarbaijan-e-Sharghi: $1 \hat{\jmath}, 1$ 을 Kaleibar, Arabshahi, $1391 \mathrm{~m}$, 38 51'42.7"N, 4708'01.1"E, 3.September.2007, leg. Mozaffarian (Fig. 1, ASh4).

Dlabola $(1971,1981)$ reported this species from Sufian, Maku and Miyaneh-Siah chaman (Fig. 1, Ash7, AG1, ASh15)

Worldwide distribution. Afro-tropical region, East Palaearctic, Europe (Austria, Balearic Is., Belgium, Britain I., Bulgaria, Canary Is., Crete, Cyprus, Greek mainland, Hungary, Italian mainland, Moldova, Portuguese mainland, Romania, South Russia, Sardinia, Sicily, Slovakia, Spanish mainland, Switzerland, Yugoslavia), Near East, North Africa (De Jong 2013).

\section{Subfamily: Aphrodinae \\ Tribe: Aphrodini}

\section{Aphrodes bicinctus (Schrank, 1776)*}

Localities. Sufian, Marand (Dlabola 1981) (Fig. 1, ASh7, ASh6).

Worldwide distribution. Europe (Albania, Austria, Belgium, Britain I., Bulgaria, Corsica, Crete, Croatia, Cyprus, Czech Republic, Danish mainland, Estonia, Finland, French mainland, Germany, Greek mainland, Hungary, Ireland, Italian mainland, Latvia, Lithuania, Madeira, Republic of Moldova, Norwegian mainland, Poland, Portuguese mainland, Romania, Russia Central, Russia North, South Russia, Sardinia, Sicily, Slovakia, Slovenia, Spanish mainland, Sweden, Switzerland, The Netherlands, Ukraine, Yugoslavia) (De Jong 2013). 


\section{Subfamily: Cicadellinae \\ Tribe: Cicadellini}

Cicadella viridis (Linnaeus, 1758)

Cicadella viridis: Le Quesne 1965: 24, fig. 115.

Material examined. Ardabil: $8 \overbrace{}^{\AA} \uparrow$, Heiran, $1527 \mathrm{~m}, 37^{\circ} 41^{\prime} 07.4^{\prime \prime} \mathrm{N}, 48^{\circ} 23^{\prime} 57.4^{\prime \prime} \mathrm{E}$, 18.January.2007, leg. Mozaffarian, Light trap (Fig. 1, A4).

Ardabil: $10^{\top}, 79,10 \mathrm{~km}$ to Parsabad, 39³6' $8.3^{\prime \prime N}$, 4748'45.5"E, 18.January. 2007, leg. Mozaffarian (Fig. 1, A2).

Azarbaijan-e-Sharghi: $25{ }^{\top}$, , Eskanlu, Aras river, $290 \mathrm{~m}, 39^{\circ} 12^{\prime} 13.4^{\prime \prime} \mathrm{N}, 47^{\circ}$ 04'23.2"E, 3.Sebtember.2007, leg. Mozaffarian \& Nematian (Fig. 1, ASh1).

Azarbaijan-e-Gharbi: 1ठ, Maku, Cheshme Soraya, 900 m, 22.August.1994, leg. Ebrahimi \& Sarafrazi (Fig. 1, AG1).

Dlabola (1981) reported this species from Zonuschay (Fig. 1, ASh5).

Worldwide distribution. East Palaearctic, Europe (Albania, Austria, Belgium, Britain I., Bulgaria, Croatia, Czech Republic, Danish mainland, Estonia, Finland, French mainland, Germany, Greek mainland, Hungary, Ireland, Italian mainland, Latvia, Lithuania, Moldova, Norwegian mainland, Poland, Romania, Russia Central, Russia North, South Russia, Sardinia, Sicily, Slovakia, Slovenia, Spanish mainland, Sweden, Switzerland, The Netherlands, Ukraine, Yugoslavia), Near East, Nearctic region, Oriental region (De Jong 2013).

Comment. Behdad (1993) reported this species as a rice pest.

\section{Subfamily: Deltocephalinae Tribe: Athysanini}

\section{Conosanus obsoletus (Kirschbaum, 1858)}

Conosanus obsoletus: Ribaut 1952: 95, 99, figs 137-138; Le Quesne 1969: 109, figs 593, 596.

Material examined. Azarbaijan-e-Gharbi: $15{ }^{\lambda}$,, Mahabad, KoushkDareh, $1499 \mathrm{~m}$, $36^{\circ} 27^{\prime} 08.6^{\prime \prime}$ N, 04542'32.9"E, 28.August.2007, leg. Mozaffarian \& Nematian (Fig. 1, AG5).

Dlabola, 1981 reported this species from Sufian and Marand. (Fig. 1, ASh7, ASh6).

Worldwide distribution. East Palaearctic, Europe (Albania, Austria, Azores, Belgium, Britain I., Bulgaria, Cyprus, Czech Republic, Danish mainland, Estonia, French mainland, Germany, Greek mainland, Hungary, Ireland, Italian mainland, Latvia, Lithuania, Moldova, Norwegian mainland, Poland, Portuguese mainland, Romania, Sicily, Slovakia, Slovenia, Spanish mainland, Sweden, Switzerland, The Netherlands, Ukraine, Yugoslavia), Near East, Nearctic region, North Africa (De Jong 2013). 


\section{Eohardya miyaneha Dlabola, 1971*}

Localities. Miyaneh- Siah chaman (Dlabola 1971) (Fig. 1, ASh15).

Worldwide distribution. Iran (Dlabola 1971).

\section{Euscelis alsius Ribaut, 1952}

Euscelis alsius: Ribaut 1952: 95, fig. 130.

Material examined. Ardabil: $20^{\wedge}+$, Moghan, 65 m, 39 $37^{\prime} 30.7^{\prime \prime} \mathrm{N}, 47^{\circ} 46^{\prime} 57.5^{\prime \prime} \mathrm{E}$, 19.January.2007, leg. Mozaffarian (Fig. 1, A1).

Ardabil: $21{ }^{\wedge}$, Parsabad, $80 \mathrm{~m}, 39^{\circ} 36^{\prime} 8.3^{\prime \prime} \mathrm{N}, 47^{\circ} 48^{\prime} 45.5^{\prime \prime} \mathrm{E}$, 18.January.2007, leg. Mozaffarian (Fig. 1, A2).

Ardabil: $10^{\lambda}, 1$, $12 \mathrm{~km}$ to Khalkhal, $1998 \mathrm{~m}, 37^{\circ} 35^{\prime} 41.8^{\prime \prime} \mathrm{N}, 48^{\circ} 37^{\prime} 54.3^{\prime \prime E}$, 17.Janaury.2007, leg. Mozaffarian, Light trap (Fig. 1, A5).

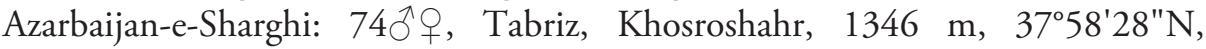
4602'55"E, 21-30.August.2007, leg. Lotfalizadeh, Malaise trap (Fig. 1, ASh8).

Azarbaijan-e-Sharghi: 10, Sahand mountain, Kandovan, 2661 m, 37 $45^{\prime} 47.7^{\prime \prime N}$,

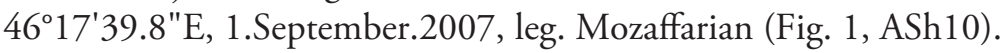

Dlabola (1981) reported this species from Zonuschay and Sufian (Fig. 1, ASh5, ASh 7).

Worldwide distribution. East Palaearctic, Europe (Bulgaria, French mainland, Greek mainland, Italian mainland, Portuguese mainland, Sicily, Spanish mainland, Yugoslavia), Near East, North Africa (De Jong 2013).

\section{Handianus bejbienkoi Dlabola, 1959}

Handianus bejbienkoi: Emeljanov 1964: 523, fig. 188: 26-27.

Material examined. Ardabil: $10^{\wedge}, 10 \mathrm{~km}$ to Parsabad, $39^{\circ} 36^{\prime} 8.3^{\prime \prime} \mathrm{N}, 47^{\circ} 48^{\prime} 45.5^{\prime \prime} \mathrm{E}$, 18.January.2007, leg. Mozaffarian (Fig. 1, A2).

Dlabola 1981 reported this species from Zonuschay and Maku (Fig. 1, ASh5, AG1).

Worldwide distribution. East Palaearctic, Europe (Ukraine), Near East (De Jong 2013).

\section{Hardya anatolica Zachvatkin, 1946*}

Localities. Marand (Dlabola 1981) (Fig. 1, ASh6).

Worldwide distribution. Europe (Bulgaria, Greek mainland, Italian mainland, Romania, Yugoslavia), Near East (De Jong 2013). 
Hardya iranicola Zachvatkin, 1946*

Localities. Sufian (Dlabola 1981) (Fig. 1, ASh7).

Worldwide distribution. Iran (Nast 1972).

Platymetopius chloroticus Puton, $1877^{*}$

Localities. Sufian, Zonuschay (Dlabola 1981) (Fig. 1, ASh7, ASh5).

Worldwide distribution. East Palaearctic, Europe (South Russia, Ukraine), Near East (De Jong 2013).

Platymetopius safavii Dlabola, 1971*

Localities. Sufian (Dlabola 1981) (Fig. 1, ASh7).

Worldwide distribution. Iran (Dlabola 1981).

Platymetopius shirazicus Dlabola, 1974*

Localities. Marand (Dlabola 1981) (Fig. 1, ASh6).

Worldwide distribution. Iran (Dlabola 1981).

\section{Tribe: Chiasmini}

Aconura jakowlefi Lethierry, 1876*

Localities. Sufian, Zonuschay (Dlabola 1981) (Fig. 1, ASh7, ASh5).

Worldwide distribution. East Palaearctic, Europe (South Russia), Near East (De Jong 2013).

Chiasmus conspurcatus (Perris, 1857)*

Localities. Miyaneh-Siah chaman (Dlabola 1971) (Fig. 1, ASh15).

Worldwide distribution. East Palaearctic, Europe (Albania, Austria (doubtful), Bulgaria, Canary Is., French mainland, Greek mainland, Italian mainland, Portuguese mainland, Romania, Sardinia, Sicily, Slovenia, Spanish mainland, Switzerland, Yugoslavia), Near East (De Jong 2013). 


\section{Doratura marandica Dlabola, 1981*}

Localities. Marand (Dlabola 1981) (Fig. 1, ASh6).

Worldwide distribution. Iran (Dlabola 1981).

\section{Doratura stylata (Boheman, 1847)}

Doratura stylata: Le Quesne 1969: 67, figs 329-331; Biedermann and Niedringhaus 2009: 298.

Material examined. Aradebil: $11 \overbrace{}^{\uparrow}+$, Sarein. Ardestan, 1700 m, 2.July.1997, leg. Barari \& Mofidi (Fig. 1, A3).

Azarbaijan-e-Sharghi: $10^{\lambda}$, Bonab, $13.6 \mathrm{~m}, 37^{\circ} 26^{\prime} 14.4^{\prime \prime} \mathrm{N}, 045^{\circ} 57^{\prime} 56.7^{\prime \prime} \mathrm{E}$, 27.August, 2007, leg. Mozaffarian \& Nematian (Fig. 1, ASh9).

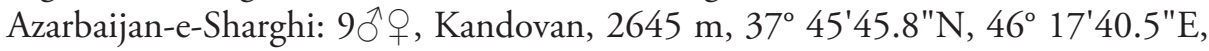
18.January.2008, leg. Mozaffarian. (Fig. 1, ASh10).

Worldwide distribution. East Palaearctic, Europe (Albania, Austria, Belgium, Britain I., Bulgaria, Czech Republic, Danish mainland, Estonia, Finland, French mainland, Germany, Greek mainland, Hungary, Italian mainland, Latvia, Lithuania, Moldova, Norwegian mainland, Poland, Portuguese mainland, Romania, Russia Central, Russia North, South Russia, Slovakia, Slovenia, Spanish mainland, Sweden, Switzerland, The Netherlands, Ukraine, Yugoslavia), Near East, Nearctic region, North Africa (De Jong 2013).

This species is newly recorded from Iran.

Doraturopsis heros (Melichar, 1902)*

Localities. Zonuschay, Marand (Dlabola 1981) (Fig. 1, ASh5, ASh6).

Worldwide distribution. Europe (South Russia, Ukraine) (De Jong 2013).

\section{Tribe: Cicadulini}

Stenometopiellus iranicus Zachvatkin, 1946*

Localities. Marand (Dlabola 1981) (Fig. 1, ASh6).

Worldwide distribution. Iran (Dlabola 1981); Uzbekistan (Nast 1972). 


\section{Tribe: Goniagnathini}

\section{Goniagnathus brevis (Herrich-Schäffer, 1835)}

Goniagnathus brevis: Emeljanov 1964: 501, fig. 180: 7, 8; Biedermann and Niedringhaus 2009: 283.

Material examined. Azarbaijan-e-Sharghi: $10^{\lambda}$, Kaleibar, $1863 \mathrm{~m}, 38^{\circ} 52^{\prime} 13.5^{\prime \prime} \mathrm{N}$, 4658'14.5"E, 2.September.2007, leg. Mozaffarian (Fig. 1, ASh3).

Abdollahi et al. (2013) reported this species from the above locality.

Worldwide distribution. East Palaearctic, Europe (Albania, Austria, Belgium, Bulgaria, Cyprus, Czech Republic, European Turkey, French mainland, Germany, Greek mainland, Hungary, Italian mainland, Moldova, Poland, Portuguese mainland, Romania, South Russia, Sicily, Slovakia, Spanish mainland, Switzerland, The Netherlands, Ukraine, Yugoslavia), Near East, North Africa (De Jong 2013).

\section{Goniagnathus guttulinervis (Kirschbaum, 1868)*}

Localities. Sufian (Dlabola 1981) (Fig. 1, ASh7).

Worldwide distribution. Afro-tropical region, East Palaearctic, Europe (Balearic Is., Canary Is., French mainland, Greek mainland, Hungary, Italian mainland, Portuguese mainland, South Russia, Sardinia, Sicily, Spanish mainland, The Netherlands (doubtful), Ukraine), Near East, North Africa (De Jong 2013).

\section{Goniagnathus minor Kusnezov, 1928*}

Localities. Miyaneh- Siah chaman (Dlabola 1971) (Fig. 1, ASh15).

Worldwide distribution. Ukraine (Nast 1972).

\section{Tribe: Hecalini}

\section{Hecalus glaucescens (Fieber, 1866)*}

Localities. Sufian (Dlabola 1981) (Fig. 1, ASh7).

Worldwide distribution. East Palaearctic, Europe (Bulgaria, Cyprus, Greek mainland, Italian mainland, South Russia, Sicily, Slovakia, Spanish mainland, Ukraine, Yugoslavia), Near East, North Africa (De Jong 2013). 


\section{Tribe: Limotettigini}

\section{Limotettix striola (Fallén, 1806)}

Limotettix striola: Emeljanov 1964: 529, fig. 190: 2-5; Biedermann and Niedringhaus 2009: 322.

Material examined. Ardabil: 1 , Moghan, Parsabad, 9.May.1969, leg. Abaii. (Fig. 1, A1) Azarbaijan-e-Sharghi: 1ㅇ, Tabriz, Gharachaman, 1600 m, 16.January.1976, leg. Boroumand \& Pazouki (Fig. 1, ASh8).

Dlabola (1981) reported this species from Sufian (Fig. 1, ASh7).

Worldwide distribution. East Palaearctic, Europe (Albania, Austria, Azores, Belgium, Britain I., Bulgaria, Cyprus, Czech Republic, Danish mainland, Estonia, Finland, French mainland, Germany, Greek mainland, Hungary, Ireland, Italian mainland, Latvia, Lithuania, Moldova, Norwegian mainland, Poland, Portuguese mainland, Romania, Russia Central, Russia North, South Russia, Slovakia, Slovenia, Spanish mainland, Sweden, Switzerland, The Netherlands, Ukraine, Yugoslavia), Near East, Nearctic region, North Africa (De Jong 2013).

\section{Tribe: Macrostelini}

\section{Balclutha flavella Linnavuori, 1962*}

Localities. Zonuschay, Marand (Dlabola 1981) (Fig. 1, ASh5, ASh6). Worldwide distribution. Israel (Nast 1972).

\section{Balclutha punctata (Fabricius, 1775)}

Balclutha punctata: Emeljanov 1964: 507, fig. 182: 3; Biedermann and Niedringhaus 2009: 286.

Material examined. Ardabil: $32{ }^{\circ}$ 우, Heiran, $1527 \mathrm{~m}, 37^{\circ} 41^{\prime} 07.4^{\prime \prime} \mathrm{N}, 48^{\circ} 23^{\prime} 57.4^{\prime \prime} \mathrm{E}$, 18.January.2007, Light trap, leg. Mozaffarian (Fig. 1, A4).

Worldwide distribution. Australian region, East Palaearctic, Europe (Albania, Austria, Britain I., Bulgaria, Cyprus, Czech Republic, Danish mainland, Estonia, Finland, French mainland, Germany, Greek mainland, Hungary, Ireland, Italian mainland, Latvia, Lithuania, Moldova, Norwegian mainland, Poland, Russia Central, Russia North, South Russia, Sardinia, Sicily, Slovakia, Sweden, Switzerland, The Netherlands, Ukraine, Yugoslavia), Near East, Nearctic region, North Africa, Oriental region (De Jong 2013).

This species is newly recorded from northwestern Iran. 


\section{Balclutha rhenana Wagner, 1939*}

Localities. Marand (Dlabola 1981) (Fig. 1, ASh6).

Worldwide distribution. East Palaearctic, Europe (Austria, Bulgaria, Czech Republic, Finland, Germany, Greek mainland (doubtful), Slovakia, Switzerland, The Netherlands, Yugoslavia) (De Jong 2013).

\section{Macrosteles chobauti Ribaut, 1952}

Macrosteles chobauti: Ribaut 1952: 48, figs 26-28.

Material examined. Azarbaijan-e-Sharghi: $51{ }^{\top}$ \%, Kandovan, $2645 \mathrm{~m}, 37^{\circ} 45^{\prime} 45.8^{\prime \prime} \mathrm{N}$, 46ำ'40.5"E, 18.January.2008, leg. Mozaffarian (Fig. 1, ASh10).

Abdollahi et al. (2014) also reported this species from the above locality.

Worldwide distribution. Europe (Bulgaria, French mainland, Greek mainland) (De Jong 2013), France, Israel (Nast 1972).

\section{Macrosteles fieberi (Edwards, 1889)*}

Localities. Sufian (Dlabola 1981) (Fig. 1, ASh7).

Worldwide distribution. East Palaearctic, Europe (Austria, Britain I., Bulgaria, Czech Republic, Finland, French mainland, Germany, Greek mainland (doubtful), Ireland, Moldova, Norwegian mainland, Poland, Romania, South Russia, Slovakia, Sweden, Switzerland, The Netherlands, Ukraine, Yugoslavia), Near East, Nearctic region (De Jong 2013).

\section{Macrosteles laevis (Ribaut, 1927)*}

Localities. Zonuschay, Maku (Dlabola 1981); Miynaeh-Zanjan, Miyaneh-Siah chaman, Tabriz-Shabestar (Dlabola 1971) (Fig. 1, ASh5, AG1, ASh16, ASh15, ASh7).

Worldwide distribution. East Palaearctic, Europe (Albania, Austria, Belgium, Britain I., Bulgaria, Czech Republic, Danish mainland, Estonia, Finland, French mainland, Germany, Greek mainland, Hungary, Iceland, Italian mainland, Latvia, Lithuania, Moldova, Norwegian mainland, Poland, Romania, Russia Central, Russia North, South Russia, Slovakia, Sweden, Switzerland, The Netherlands, Ukraine, Yugoslavia), Near East, Nearctic region (De Jong 2013).

Comment. Kheyri (1989) reported this species as a sugar beet pest in most sugar beet growing areas in Iran. 


\section{Macrosteles sexnotatus (Fallén, 1806)}

Macrosteles sexnotatus: Emeljanov 1964: 507, fig. 182: 26, 27; Biedermann and Niedringhaus 2009: 288.

Material examined. Azarbaijan-e-Sharghi: 30^ึ , Sahand mountain, Kandovan, $2661 \mathrm{~m}$, 3745'47.7"N, 46¹7'39.8"E, 1.September.2007, leg. Mozaffarian (Fig. 1, ASh10).

Abdollahi et al. (2013) reported this species from the above locality.

Worldwide distribution. East Palaearctic, Europe (Austria, Azores, Belgium, Britain I., Bulgaria, Canary Is., Cyprus, Czech Republic, Danish mainland, Estonia, Finland, French mainland, Germany, Greek mainland, Hungary, Iceland, Ireland, Italian mainland, Latvia, Lithuania, Madeira, Moldova, Norwegian mainland, Poland, Portuguese mainland, Romania, Russia Central, South Russia, Sardinia, Sicily, Slovakia, Spanish mainland, Sweden, Switzerland, The Netherlands, Ukraine, Yugoslavia), Near East, North Africa (De Jong 2013).

\section{Macrosteles sordidipennis (Stål, 1858)}

Macrosteles sordidipennis: Emeljanov 1964: 507, fig. 182: 36, 37; Biedermann and Niedringhaus 2009: 290.

Material examined. Azarbaijan-e-Sharghi: 10, Sahand Mountain, Kandovan, 2661 m, 37'45'47.7"N, 46²17'39.8"E, 1.September.2007, leg. Mozaffarian (Fig. 1, ASh10).

Worldwide distribution. East Palaearctic, Europe (Austria, Britain I., Czech Republic, Danish mainland, Finland, Germany, Hungary, Norwegian mainland, Poland, Russia North, Sweden, The Netherlands) (De Jong 2013).

This species is newly recorded from Iran.

\section{Tribe: Opsiini}

\section{Concavifer marmoratus Dlabola, 1960*}

Localities. Zonuschay (Dlabola 1981) (Fig. 1, ASh5).

Worldwide distribution. Iran, Israel, Kazakhstan, Tadzhikistan (Nast 1972).

\section{Neoaliturus fenestratus (Herrich-Schäffer, 1834)*}

Localities. Tabriz (Dlabola 1981) (Fig. 1, ASh8).

Worldwide distribution. East Palaearctic, Europe (Albania, Austria, Balearic Is., Belgium, Bulgaria, Canary Is., Cyprus, Czech Republic, Danish mainland, European Tur- 
key, French mainland, Germany, Greek mainland, Hungary, Italian mainland, Latvia, Lithuania, Moldova, Poland (doubtful), Portuguese mainland, Romania, Russia Central, Russia North, South Russia, Sardinia, Sicily, Slovakia, Spanish mainland, Switzerland, The Netherlands, Ukraine, Yugoslavia), Near East, North Africa (De Jong 2013).

\section{Neoaliturus haematoceps (Mulsant Rey, 1855)*}

Localities. Marand, Zonuschay, Sufian, Maku (Dlabola 1981) (Fig. 1, ASh6, ASh5, ASh7, AG1).

Worldwide distribution. Afghanistan, Algeria, Austria, Canary Is., Cyprus, Czechoslovakia (Bohemia, Moravia, Slovakia), Egypt, France, German FR., Greece, Hungary, Iran, Italy (also Sardinia and Sicily), Jordan, Lebanon, Libya, Madeira Archipelago, Mongolia, Morocco, Poland, Romania, Spain, Syria, Tunisia, Turkey (Anatolia), Armenia, Azerbaijan, Georgia, Kazakhstan, Kirghizia, Moldavia, s.Russia, Turkmenia, Ukraine, Uzbekistan, Yugoslavia (Nast 1972).

Comment. Kheyri (1989) reported this species as an economic pest on sugar beet from Isfahan, Kerman, Fars, Khorasan, Azarbaijan and Karaj.

\section{Neoaliturus opacipennis (Lethierry, 1876)*}

Localities. Miyaneh-Zanjan, Bostanabad-Siah chaman, Siah chaman-Basmenj (Dlabola 1971) (Fig. 1, ASh16, ASh14, ASh13).

Worldwide distribution. Europe (Cyprus, French mainland, Germany, Greek mainland, Italian mainland, South Russia, Sardinia, Sicily, Spanish mainland, Switzerland, Ukraine), Near East, North Africa (De Jong 2013).

Comment. Kheyri and Alimoradi (1969) reported this species as a vector of curly top virus in Khorasan, Fars, Isfehan, Kerman, Ahvaz and Karaj.

Neoaliturus pulcher (Haupt, 1927)*

Localities. Zonuschay (Dlabola 1981) (Fig. 1, ASh5).

Worldwide distribution. Iran, Israel, Georgia, Kazakhstan, Tadzhikistan (Nast 1972).

\section{Opsius cypriacus Lindberg, 1958*}

Localities. Zonuschay (Dlabola 1981) (Fig. 1, ASh5).

Worldwide distribution. Europe (Cyprus, Greek mainland, Ukraine), Near East (De Jong 2013). 
Opsius discessus (Horváth, 1911)*

Localities. Zonuschay, Marand (Dlabola 1981) (Fig. 1, ASh5, ASh6).

Worldwide distribution. East Palaearctic, Europe (South Russia), Near East (De Jong 2013).

\section{Opsius pallasi (Lethierry, 1874)*}

Localities. Zonuschay, Marand (Dlabola 1981) (Fig. 1, ASh5, ASh6).

Worldwide distribution. Europe (South Russia) (De Jong 2013).

\section{Opsius scutellaris (Lethierry, 1874)*}

Localities. Zonuschay (Dlabola 1981) (Fig. 1, ASh5).

Worldwide distribution. Afro-tropical region, East Palaearctic, Europe (Canary Is.), Near East, North Africa (De Jong 2013).

Pseudophlepsius binotatus (Signoret, 1880)*

Localities. Zonuschay, Sufian (Dlabola 1981) (Fig. 1, ASh5, ASh7).

Worldwide distribution. Europe (South Russia) (De Jong 2013).

\section{Tribe: Paralimnini}

Mogangella straminea Dlabola, 1957

Mogangella straminea: Emeljanov 1964: 541, fig. 194: 13, 14.

Material examined. Azarbaijan-e-Sharghi: $1 \delta 1$ 1 , Marand, 12.July.2007, Light trap, leg. Lotfalizadeh (Fig. 1, ASh6).

Worldwide distribution. East Palaearctic, Europe (Moldova, Ukraine), Near East (De Jong 2013).

This species is newly recorded from Iran.

Paramesus major Haupt, 1927*

Localities. Sufian (Dlabola 1981) (Fig. 1, ASh7). 
Worldwide distribution. East Palaearctic, Europe (Austria, Bulgaria, Czech Republic, Germany (doubtful), Greek mainland, Hungary, Poland, South Russia, Yugoslavia), Near East (De Jong 2013).

\section{Paramesus paludosus Ribaut, 1952*}

Localities. Sufian (Dlabola 1981) (Fig. 1, ASh7).

Worldwide distribution. France, Italy, Kazakhstan, Moldavia, Ukraine (Nast 1972).

\section{Psammotettix alienus (Dahlbom, 1850)}

Psammotettix alienus: Ribaut 1952: 243, figs 579-580; Emeljanov 1964: 541, fig. 194: 8, 9; Biedermann and Niedringhaus 2009: 337.

Material examined. Ardabil: $4 \widehat{\wp}+$, Moghan, 18.May.1978, leg. Abaii (Fig. 1, A1). Azarbaijan-e-Sharghi: 3ठํ , Marand, 1610 m, 28.July.1976, leg. Broumand \& Pazouki (Fig. 1, ASh6).

Azarbaijan-e-Sharghi: 1ㅇ, Tabriz, Gharachaman, 1600 m, 16.July.1976, leg. Broumand \& Pazouki (Fig. 1, ASh8).

Dlabola (1981) reported this species from Marand, Sufian and Zonuschay, Maku and in 1971 from Tabriz-Bostanabad, Miyaneh-Zanjan, Miyaneh-Siah chaman, Siah chaman-Basmenj (Fig. 1, ASh6, ASh7, ASh5, AG1, ASh12, ASh16, ASh15, ASh13).

Worldwide distribution. East Palaearctic, Europe (Albania, Austria, Belgium, Bulgaria, Canary Is., Czech Republic, Danish mainland, Estonia, Finland, French mainland, Germany, Greek mainland, Hungary, Italian mainland, Latvia, Lithuania, Madeira, Moldova, Norwegian mainland, Poland, Portuguese mainland, Romania, Russia Central, Russia North, South Russia, Sicily, Slovakia, Slovenia, Spanish mainland, Sweden, Switzerland, Ukraine, Yugoslavia), Near East, Nearctic region, North Africa (De Jong 2013).

Comment. Nematollahi and Khajehali (2000) reported this species as a vector for wheat dwarf virus on Zea (maize) in Isfahan.

\section{Psammotettix pictipennis (Kirschbaum, 1868)*}

Localities. Miyaneh-Zanjan (Dlabola 1971); Marand, Sufian (Dlabola 1981) (Fig. 1, ASh16, ASh6, ASh7).

Worldwide distribution. East Palaearctic, Europe (Austria, Bulgaria, Greek mainland, Hungary, Moldova, Romania, South Russia, Slovenia, Spanish mainland, Ukraine, Yugoslavia), Near East (De Jong 2013). 


\section{Psammotettix seriphidii Emeljanov, 1962}

Psammotettix seriphidii: Emeljanov 1964: 539, fig. 193: 1, 2.

Material examined. Ardabil: $87 \delta^{\wedge}$ ㅇ, $12 \mathrm{~km}$ to Khalkhal, $1998 \mathrm{~m}, 37^{\circ} 35^{\prime} 41.8^{\prime \prime} \mathrm{N}$, 483'ㄴ.3"E, 17.Janaury.2007, leg. Mozaffarian, light trap (Fig. 1, A5).

Ardabil: $10^{\lambda}, 10 \mathrm{~km}$ to Parsabad, $80 \mathrm{~m}, 39^{\circ} 36^{\prime} 8.3^{\prime \prime} \mathrm{N}, 47^{\circ} 48^{\prime} 45.5^{\prime \prime E}, 18$.January.2007, leg. Mozaffarian (Fig. 1, A2).

Ardabil: $3{ }^{\Uparrow}+$, Moghan, 65 m, 39³ $37^{\prime} 30.7^{\prime \prime} \mathrm{N}, 47^{\circ} 46^{\prime} 57.5^{\prime \prime} \mathrm{E}$, 19.January.2007, leg. Mozaffarian (Fig. 1, A1).

Azarbaijan-e-Sharghi: $60^{\wedge}$ 을, AjabShir, Yaichi village, $1922 \mathrm{~m}, 37^{\circ} 35^{\prime} 27.2^{\prime \prime} \mathrm{N}$, 46¹1'03.7"E, 15.January.2008, leg. Mozaffarian (Fig. 1, ASh11).

Azarbaijan-e-Sharghi: $2{ }^{\top} \%$, Kaleibar, $1732 \mathrm{~m}, 38^{\circ} 54^{\prime} 25.2^{\prime \prime N}, 47^{\circ} 09^{\prime} 11.5^{\prime \prime E}$, 3.September.2007, leg. Mozaffarian (Fig. 1, ASh2).

Azarbaijan-e-Sharghi: $10^{\top}$, Kandovan, $2645 \mathrm{~m}, 37^{\circ} 45^{\prime} 45.8^{\prime \prime} \mathrm{N}, 46^{\circ} 17^{\prime} 40.5^{\prime \prime} \mathrm{E}$, 18.January.2008, leg. Mozaffarian (Fig. 1, ASh10).

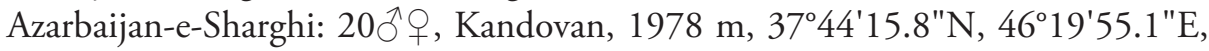
18.January.2008, leg. Mozaffarian (Fig. 1, ASh10).

Azarbaijan-e-Sharghi: 40ㅇ, Eskanlu, Aras river, 290 m, 39¹2'13.4"N, 47 04'23.2"E, 3.September.2007, leg. Mozaffarian \& Nematian (Fig. 1, ASh1).

Azarbaijan-e-Gharbi: 11 $\overbrace{}^{\lambda}$, Uromieh, MirzaAbad, 1450 m, 21.July.1976, leg. Boroumand \& Pazouki (Fig. 1, AG4).

Worldwide distribution. Kazakhstan (Nast 1972).

This species is newly recorded from Iran.

\section{Sorhoanus medius (Mulsant Rey, 1855)*}

Localities. Sufian (Dlabola 1981) (Fig. 1, ASh7)

Worldwide distribution. Bulgaria, France, Italy, Switzerland, Altai Mts., Kazakhstan, Kirghizia, Russia, Siberia, Ukraine, Yugoslavia (Nast 1972).

\section{Tribe: Phlepsiini}

\section{Phlepsius intricatus (Herrich-Schäffer, 1838)}

Phlepsius intricatus: Emeljanov 1964: 516, fig. 185: 5, 6; Biedermann and Niedringhaus 2009: 305.

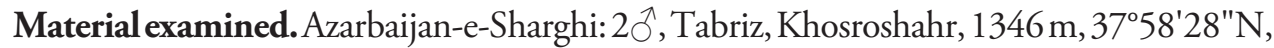
$46^{\circ} 02^{\prime} 55^{\prime} \mathrm{E}, 21-30$.August.2007, leg. Lotfalizadeh, Malaise trap (Fig. 1, ASh8).

Dlabola (1981) reported this species from Zonuschay and in 1974 from Uromieh (Fig. 1, ASh5, AG3). 
Worldwide distribution. Europe (Albania, Austria, Balearic Is., Bulgaria, Czech Republic, European Turkey, French mainland, Germany, Greek mainland, Hungary, Italian mainland, Moldova, Portuguese mainland, Romania, South Russia, Sardinia, Sicily, Slovakia, Slovenia, Spanish mainland), Near East, North Africa (De Jong 2013).

\section{Tribe: Scaphoideini}

Anoplotettix magnificus Emeljanov, 1962 *

Localities. Sufian (Dlabola 1981) (Fig. 1, ASh7).

Worldwide distribution. Azarbaijan, Georgia (Nast 1972).

\section{Subfamily: Dorycephalinae}

Tribe: Eupelicini

Eupelix cuspidata (Fabricius, 1775)

Eupelix cuspidata: Ribaut 1952: 325, figs 868-871.

Material examined. Azaibaijan-e-Sharghi: 4^우 Ajabshir, Yaichi village, $1922 \mathrm{~m}$, 37³5'27.2"N, 46¹1'03.7"E, 15.January.2008, leg. Mozaffarian (Fig. 1, ASh11).

Dlabola (1981) reported this species from Zonuschay, Marand and Sufian (Fig. 1, ASh5, ASh6, ASh7).

Worldwide distribution. East Palaearctic, Europe (Albania, Austria, Balearic Is., Belgium, Britain I., Bulgaria, Canary Is., Corsica, Croatia, Cyprus, Czech Republic, Danish mainland, Estonia, Finland, French mainland, Germany, Greek mainland, Hungary, Ireland, Italian mainland, Latvia, Lithuania, Moldova, Norwegian mainland, Poland, Portuguese mainland, Romania, Russia Central, Russia North, South Russia, Sardinia, Sicily, Slovakia, Slovenia, Spanish mainland, Sweden, Switzerland, The Netherlands, Ukraine, Yugoslavia), Near East, North Africa (De Jong 2013).

Paradorydium aristidae (Zachvatkin, 1953)*

Localities. Zonuschay, Maku (Dlabola 1981) (Fig. 1, ASh5, AG1).

Worldwide distribution. East Palaearctic, Europe (South Russia, Ukraine), Near East (De Jong 2013).

Subfamily: Iassinae

Tribe: Iassini 


\section{Batracomorphus irroratus Lewis, 1834*}

Localities. Marand (Dlabola 1981) (Fig. 1, ASh6).

Worldwide distribution. East Palaearctic, Europe (Albania, Austria, Belgium, Britain I., Bulgaria, Czech Republic, Danish mainland, French mainland, Germany, Greek mainland, Hungary, Italian mainland, Lithuania, Moldova, Poland, South Russia, Slovakia, Switzerland, Ukraine, Yugoslavia), Near East (De Jong 2013).

\section{Subfamily: Idiocerinae}

Tribe: Idiocerini

Rhytidodus resaicus Dlabola, 1974*

Localities. Uromieh (Dlabola 1974) (Fig. 1, AG3)

\section{Subfamily: Macropsinae}

Tribe: Macropsini

Hephathus unicolor (Lindberg, 1926)*

Localities. Zonuschay (Dlabola 1981) (Fig. 1, ASh5).

Worldwide distribution. East Palaearctic, Europe (Romania (doubtful), South Russia (doubtful), Ukraine (doubtful), Yugoslavia (doubtful)), Near East (De Jong 2013).

\section{Hephathus freyi (Fieber, 1868)*}

Localities. Siah chaman-Miyaneh (Dlabola 1971) (Fig. 1, ASh15).

Worldwide distribution. Europe (Balearic Is., Bulgaria, French mainland, Greek mainland (doubtful), Italian mainland, Portuguese mainland, Sicily, Slovakia, Spanish mainland, Yugoslavia), Near East, North Africa (De Jong 2013).

\section{Subfamily: Typhlocybinae}

Tribe: Empoascini

Empoasca punjabensis Singh-Pruthi, 1940*

Localities. Zonuschay, Maku (Dlabola 1981); Siah chaman-Miyaneh, MiyanehZanjan, Tabriz-Shabestar, Uromieh-Sarv (Dlabola 1971) (Fig. 1, ASh5, AG1, ASh15, ASh16, ASh7, AG2). 
Worldwide distribution. Europe (Bulgaria, French mainland, Greek mainland, South Russia, Ukraine, Yugoslavia), Near East, Oriental region (De Jong 2013).

Comment. Kheyri (1989) reported this species as an economic pest on sugar beet from Isfahan, Kerman, Fars and Karaj.

\section{Kyboasca bipunctata (Oshanim, 1871)*}

Localities. Miyaneh- Siah chaman, Tabriz-Shabestar, Miyaneh-Zanjan (Dlabola 1971); Sufian (Dlabola 1981) (Fig. 1, ASh15, ASh7, ASh16, ASh7).

Worldwide distribution. East Palaearctic, Europe (Austria, Bulgaria, Czech Republic, Danish mainland, Finland, Germany, Hungary, Italian mainland, Moldova, Poland, Romania, South Russia, The Netherlands, Ukraine, Yugoslavia), Near East, Nearctic region (De Jong 2013).

\section{Tribe: Erythroneurini}

\section{Tamaricella ribauti (Zachvatkin, 1947)*}

Localities. Zonuschay (Dlabola 1981) (Fig. 1, ASh5).

Worldwide distribution. Europe (Crete, South Russia, Ukraine) (De Jong 2013).

\section{Tamaricella tamaricis (Puton, 1872)*}

Localities. Miyaneh-Zanjan, Miyaneh-Siah chaman (Dlabola 1971) (Fig. 1, ASh16, ASh15).

Worldwide distribution. Europe (Bulgaria, Crete, Cyclades Is., Cyprus, French mainland, Greek mainland, Italian mainland, Romania, South Russia, Sardinia, Sicily, Spanish mainland, Ukraine) (De Jong 2013).

\section{Zyginidia pullula (Boheman, 1845)*}

Localities. Marand (Dlabola 1981) (Fig. 1, ASh6).

Worldwide distribution. Europe (Albania, Austria, Bulgaria, Corsica (doubtful), Czech Republic, Danish mainland, Finland, French mainland, Germany, Greek mainland, Hungary, Italian mainland, Romania, Slovakia, Spanish mainland, Sweden, Switzerland, Ukraine, Yugoslavia), Near East (De Jong 2013). 


\section{Zyginidia sohrab Zachvatkin, 1947*}

Localities. Miyaneh-Zanjan, Siah chaman-Miyaneh, Uromieh-Sarv (Dlabola 1971) (Fig. 1, ASh16, ASh15, AG2).

Worldwide distribution. Europe (Cyprus, Greek mainland, South Russia, Ukraine), Near East (De Jong 2013).

\section{Tribe: Typhlocybini}

\section{Edwardsiana rosae (Linné, 1758)*}

Localities. Siah chaman-Miyaneh (Dlabola 1971) (Fig. 1, ASh15).

Worldwide distribution. East Palaearctic, Europe (Austria, Belgium, Britain I., Bulgaria, Cyprus, Czech Republic, Danish mainland, Estonia, Finland, French mainland, Germany, Greek mainland, Hungary, Ireland, Italian mainland, Latvia, Moldova, Norwegian mainland, Poland, Romania, Russia Central, Russia North, South Russia, Sicily, Slovakia, Spanish mainland, Sweden, Switzerland, The Netherlands, Ukraine, Yugoslavia), Near East, Nearctic region, Oriental region (De Jong 2013).

\section{Subfamily: Ulopinae \\ Tribe: Ulopini}

\section{Ulopa trivia Germar, 1821*}

Localities. Marand (Dlabola 1981) (Fig. 1, ASh6).

Worldwide distribution. Albania, Austria, Belgium, Bulgaria, Cyprus, Czechoslovakia (Bohemia, Moravia, Slovakia), Denmark, France, German DR, German FR, Great Britain (England), Greece, Hungary, Italy, Palestine, Poland, Portugal, Romania, Spain, Turkey (Anatolia), Azerbaijan, Armenia, Georgia, Moldavia, Russia, Ukraine, Yugoslavia (Nast 1972).

\section{Acknowledgements}

The research was performed in the Iranian Research Institute of Plant Protection using the facilities and specimens in the Insect Taxonomy Research Department and Hayk Mirzayans Insect Museum. We would like to acknowledge the assistance given by Prof. Hani Abdul-Nour, who passed away in October 2014 and Dr Paul H. Freytag (University of Kentucky, USA) for correcting and confirming some of the identifications. 


\section{References}

Abdollahi T, Jalili-Zand AR, Mozaffarian F (2014) The first report of Macrosteles chobauti (Hem., Cicadellidae) from Iran. Journal of Entomological Society of Iran 34(2): 27-28.

Abdollahi T, Mozaffarian F, Jalili-Zand AR (2013) Key to the subfamilies of Cicadellidae (Hem.: Auchenorryncha) in East Azarbaijan province, Iran and two new records for the region. Iranian Journal of Applied Plamt Protection 2(2): 147-155.

Aghagoli-Marzijarani N, Mozaffarian F, Vafaei Shushtari R (2010) Investigations on the morphological variation of populations of grape cicada Cicadatra alhageos (Kolenati, 1857) on wheat fields and vinyards using geometric morphometric methid. In: Manzari S (Ed.) Proceeding of the nineteenth Iranian Plant Protection Congress (Tehran), July-Auguast 2010. Published by Iranian Institute of Plant Protection, Tehran, 151 pp.

Aghagoli-Marzijarani N, Mozaffarian F, Vafaei Shushtari R (2013) Using wing geometric morphometric in identification of three species of grape cicads (Hem., Cicadidae) in Iran. Journal of Entomological Research 5(1): 1-10.

Behdad E (1993) Pests of field crops in Iran. Neshat Publisher, Esfehan, 618 pp.

Biedermann R, Niedringhaus R (2009) The plant and Leafhoppers of Germany. Identification key to all species. Wilhelm Brüggemann GmbH, Bremen, 409 pp.

De Jong YSDM (Ed.) (2013) Fauna Europaea (vers. 2.6.2). Web Service. http://www.faunaeur.org

Dewey JF, Hempton MR, Kidd WSF, Saroglu F, Sengor AMC (1986) Shortening of continental lithosphere: the neotectonics of Eastern Anatolia - a young collision zone. In: Coward MP, Ries AC (Eds) Collision Tectonics, Geological Society special publications 19: 3-36.

Dietrich CH (2005) Keys to the families of Cicadomorpha and subfamilies and tribes of Cicadellidae (Hemiptera, Auchenorrhyncha). Florida Entomologist 88(4): 502-517. doi: 10.1653/0015-4040(2005)88[502:KTTFOC]2.0.CO;2

Dlabola J (1971) Taxonomische und Chorologische Ergängen der Zikadenfauna von Anatolien, Iran, Afghanistan und Pakistan (Homoptera, Auchenorrhyncha). Acta Entomologica Bohemoslovaca 68: 377-396.

Dlabola J (1974) Ergebnisse der Tschechoslowakisch- Iranischen Entomologischen expedition nach dem Iran, 1970, No. 3 (Homoptera, Auchenorrhyncha) (1 Teil). Acta Faunistica Entomologica Musei Nationalis Pragae, Supplement 6: 29-73.

Dlabola J (1977) Die Tropiduchiden - Gattung Kazerunia in Iran (Homoptera: Auchenorrhyncha). Reichenbachia (Band, 16) 15: 163-167.

Dlabola J (1980) Neue Zikadenarten der Gattungen aus Sudeuropa und 6 Neue Mycterodus aus Iran (Homoptera, Auchenorrhyncha). Acta Faunistica Entomologica Musei Nationalis Pragae 16(184): 55-84.

Dlabola J (1981) Ergebnisse der tschechoslowakisch - Iranischen entomologischen expeditionen nach dem Iran (1970 und 1973) (Mit Angaben uber einige Sammelresultate in Anatolien) Homoptera, Auchenorrhyncha (II. Teil). Acta Entomologica Musei Nationalis Pragae 40: 127-311.

Dlabola J (1982) Forzetzung der Ergazungen zur Issiden - Taxonomie von Anatolien, Iran und Griechenland (Homoptera, Auchenorrhyncha). Acta Faunistica Entomologica Musei Nationalis Pragae 38 B (3): 113-168. 
Dlabola J (1983) Ergebnisse der tschecholosvakisch - Iranischen entomologischen expeditionen 1970 und 1973 nach dem Iran, neue eremische Ricaniden und Flatiden (Homoptera, Auchenorrhyncha). Acta Faunistica Entomologica Musei Nationalis Pragae 41: 91-97.

Dlabola J (1985) Neue Cixiiden vom Iran, Nach barlandern und anderen mediterangebieten (Homoptera, Auchenorrhyncha). Acta Entomologica Bohemoslovaca 82: 92-128.

Emeljanov AF (1974) Proposals on the Classification and Nomenclature of Ranges. Entomological Review 53(3): 11-26.

Emeljanov AF (1997) New species and new data on the distribution of the subfamily Orgeriinae in Palaearctic region (Homoptera, Dictyopharidae), Zoologist. Rossica 6: 83-90.

Firouz E (2005) The Complete Fauna of Iran. IB Tauris, London \& New York, 322 pp.

Gardenhire RQ (1959) Summary of insect conditions in Iran. Entomologie et Phytopathologie Appliquees 18: 51-62.

Gharib AR (1966) Ommatissus binotatus Fieb. var. lybicus Berg. (Homoptera, Tropiduchidae). Applied Entomology and Phytopathology 24: 37-47.

Gnezdilov VM, Mozaffarian F (2011) A new species of the genus Eusarima (Hemiptera, Fulgoroidea: Issidae) from Iran. Acta Entomologica Musei Nationalis Pragae 51(2): 457-462.

Haghshenas A, Khajehali J (2000) Leafhoppers (Homoptera, Auchenorrhyncha) fauna of potato fields in Chahar Mahal and Bakhtiari province. Proceeding of the fourteenth Iranian Plant Protection Congress (Isfahan), September 2000. Published by Isfahan University of Technology, Isfahan, 249 pp.

Hedge IC, Wendelbo P (1978) Patterns of distribution and endemism in Iran. Notes from the Royal Botanic Garden Edinburgh 36: 441-464.

Karimzadeh J, Kharrazi pakdel A, Kheuri M (1998) Leaf hopper (Homoptera, Auchenorrhyncha) fauna of sugarbeet fields in Isfahan province. In: Proceeding of the thirteenth Iranian Plant Protection Congress (Karaj), August 1998. Published by Karaj Junior College of Agriculture, Karaj, 69 pp.

Khajehali J, Seyedoleslami H, Kamali K (2001) A contribution to study of hoppers (Hom., Auchenorrhyncha) of potato fields in Isfahan and Daran. Applied Entomology and Phytopathology 68(1/2): 43-71.

Kheyri M (1989) An inventory of pests attacking sugar-beet in Iran. Applied Entomology and Phytopathology 56(1): 75-91.

Kheyri M, Alimoradi I (1969) The leafhoppers of sugar beet in Iran and their role in curly-top virus disease. Sugar beet seed institute, Karaj. Entomology Research Division, 50 pp.

Larivière MC, Fletcher MJ, Larochelle A (2010) Auchenorrhyncha (Insecta, Hemiptera): catalogue. Fauna of New Zealand 63: 1-232.

Lashkari M, Nouri Ganbalani G, Mozaffarian F, Ghorbani Kh, Fathi A (2009) Faunistic study of planthoppers infraorder Fulgoromorpha (Hem., Auchenorrhyncha) in different climatic regions of Gorgan, Iran. Journal of Entomological Research 1(2): 119-133.

Le Quesne WJ (1965) Handbooks for the identification of British insects Hemiptera - Homoptera: Cicadomorpha, (xcluding Deltocephalinae and Typhlocybinae), Vol. 2, Part 2(a), 64 pp.

Le Quesne WJ (1969) Handbooks for the identification of British insects Hemiptera (Cicadomorpha - Deltocephalinae), Vol. 2, Part 2(b), 65-148. 
Metcalf ZP (1962-1968) General Catalog of the Homoptera. USDA, Agric, Res. Serv. Fasc., 6, Cicadelloidea. [Part 1: Tettigellidae, 730 pp (1965); Part 2: Hylicidae, 18 pp (1962); Part 3: Gyponidae, 299 pp (1962); Part 4: Ledridae, 147 pp (1962); Part 5: Ulopidae, 101 pp (1962); Part 6: Evancanthidae, 63 pp (1963); Part 7: Nirvanidae, 35 pp (1963); Part 8: Aphrodidae, 268 pp (1963); Part 9: Hecalidae, 123 pp (1963); Part 10: Euscelidae, Section 1, 1-1077; Section 2, 1078-2074; Section 3, 2075-2695 (1967); Part 11: Coelidiidae, 182 pp (1964); Part 12: Eurymelidae, 43 pp (1965); Part 13: Macropsidae, 261 pp (1966); Part 14: Agallidae, 173 pp (1966); Part 15: Iassidae, 229 pp (1966); Part 16: Idioceridae, 237 pp (1966); Part 17: Cicadellidae, 1513 pp (1968)]

Moosavi N, Sadeghi Namaghi H (2012) Biodiversity of Auchenorrhyncha in Sugar beet fields of Mashhad region and new records for Khorasan Razavi province. Sugar beet Journal 28(1): 13-24.

Mozaffarian F, Sanborn AF, Phillips PK (2010) Cicadatra lorestanica, a new species of cicada from Iran (Hemiptera: Cicadidae). Journal of Entomological and Acarological Research Ser. II, 42(1): 27-37. doi: 10.4081/jear.2010.27

Mozaffarian F (2012a) Investigations on the fauna of planthoppers (Hemiptera: Auchenorrhyncha: Fulgoroidea) in North Western Iran. In: Sarafrazi A, Abdollahi T, Solhjouy Fard S (Eds) Proceeding of the twentieth Iranian Plant Protection Congress (Shiraz), August 2012. Published by Shiraz University, Shiraz, 133.

Mozaffarian F (2012b) A preliminary study on the distribution patterns of endemic apecies of Fulgoromorpha (Hemiptera, Auchenorrhyncha) in Iran. Sixth European Hemiptera Congress, Blagovgrad, Bulgaria, 25-29 June.

Mozaffarian F (2013) A preliminary study on the distribution patterns of endemic species of Fulgoromorpha (Hemiptera, Auchenorrhyncha) in Iran. Zookeys 319: 231-248. doi: 10.3897/zookeys.319.4159

Mozaffarian F, Emeljanov AF (2010) A faunestic study of the subfamily Dictyopharinae Spinola, 1839 (Hem., Dictyopharidae) in Iran. In: Manzari S (Ed.) Proceeding of the nineteenth Iranian Plant Protection Congress (Tehran), July-Auguast 2010. Published by Iranian Institute of Plant Protection, Tehran, 128.

Mozaffarian F, Gnezdilov VM (2011) First record of Scorlupella montana (Becker) (Hemiptera: Fulgoroidea: Issidae) from Iran. Journal of Entomological Society of Iran 30(2): 93-95.

Mozaffarian F, Taghizadeh M (2010) The first report of three leafhoppers and planthoppers from central parts (Tehran, Semnan, Ghom, Ghazvin and Markazi provinces) of Iran. In: Manzari S (Ed.) Proceeding of the nineteenth Iranian Plant Protection Congress (Tehran), July-Auguast 2010. Published by Iranian Institute of Plant Protection, Tehran, 129.

Mozaffarian F, Sanborn AF (2010) The cicadas of Iran with the description of two new species (Hemiptera, Cicadidae). Mitteilungen aus dem Museum für Naturkunde in Berlin - Deutsche Entomologische Zeitschrift 57(1): 69-84.

Mozaffarian F, Sanborn AF (2012) Two new species Tettigetta Kolenati from Iran. Zoology in the Middle East 57: 119-126. doi: 10.1080/09397140.2012.10648971

Mozaffarian F, Sanborn AF (2013) A new species of the genus Cicadatra from Iran (Hemiptera, Auchenorrhyncha, Cicadidae). Acta Entomologica Musei Nationalis Pragae 53(1): 39-48. 
Mozaffarian F, Wilson MR (2011) An annotated checklist of the planthoppers of Iran (Hemiptera, Auchenorrhyncha, Fulgoromorpha) with distribution data. Zookeys 145: 1-57. doi: 10.3897/zookeys. 145.1846

Nast J (1972) Palaearctic Auchenorrhyncha (Homoptera) An annoted check list. Polish Scientific Publishers, Warsaw, Poland, 550 pp.

Nematollahi MR, Khajehali J (2000) Major leafhoppers and planthoppers (Hom.: Auchenorrhyncha) of corn fields in Esfahan. In: Proceeding of the fourteenth Iranian Plant Protection Congress (Isfahan), September 2000. Published by Isfahan University of Technology, Isfahan, 235.

Nickel H (2003) The leafhoppers and planthoppers of Germany (Hemiptera, Auchenorrhyncha): patterns and strategies in a highly diverse group of phytophagous insects. Pensoft Publishers, Sofia-Moscow, Goecke \& Evers, Keltern, 460 pp.

Oman PW, Knight WJ, Nielson MW (1990) Leafhoppers (Cicadellidae): a biblio-graphy, generic check-list and index to the world literature 1956-1985. CAB International Institute of Entomology, Wallingford, 368 pp.

Rajabi A, Mirzayans H (1989) First report of Zyginella pulchra Low as a harmful insect on deciduous fruit trees in Iran. Applied Entomology and Phytopathology 65(1\&2): 101-103.

Ribaut H (1952) Homoptéres Auchénorhynques, II (Jassidae). Faune de France 57: 1-474.

Taghizadeh M (2012) First report of Amrasca biguttula biguttula (Ishida, 1913) (=Empoasca devastans Distant, 1918) (Order Hemiptera, Cicadellidae, Typhlocybinae) from Zarrindasht/Fars/Iran cotton fields. In: Sarafrazi A, Abdollahi T, Solhjouy Fard S (Eds) Proceeding of the twentieth Iranian Plant Protection Congress (Shiraz), August 2012. Published by Shiraz University, Shiraz, 107.

Taghizadeh M, Salehi M, Faghih MM (2010) First report of Orosius cellulosus (Lindberg) from Iran. In: Manzari S (Ed.) Proceeding of the nineteenth Iranian Plant Protection Congress (Tehran), July-Auguast 2010. Published by Iranian Institute of Plant Protection, Tehran, 141.

Tipping C, Mizell III RF, Andersen PC (2004) Dispersal adaptations of immature stages of three species of leafhopper (Hemiptera: Auchenorrhyncha: Cicadellidae). Florida Entomologist 87: 372-379. doi: 10.1653/0015-4040(2004)087[0372:DAOISO]2.0.CO;2

Weintraub PG, Beanland L (2006) Insect vectors of phytoplasmas. Annual Review of Entomology 51: 91-111. doi: 10.1146/annurev.ento.51.110104.151039

Yarmand H, Sadeghi SE, Mohammadi M, Ebrahimi SS, Seif Allahi AR (2006) Survey of arthropods associated with range medicinal plants in Tehran, Ghazvin and Isfahan provinces. Iranian Journal of Forest and Range Protection Research 3(2): 107-127.

Zarudny NA (1911) Verzeichnis der Vögel Persiens. Journal für Ornithologie 59: 185-241. doi: 10.1007/BF02091053

Zahniser JN, Dietrich CH (2013) A review of the tribes of Deltocephalinae (Hemiptera: Auchenorrhyncha: Cicadellidae). European Journal of Entomology 45: 1-211.

Zohdi H, Taghizadeh M, Mardi M (2012) Determine the dominant species leafhoppers citrus orchards in Kerman province. In: Sarafrazi A, Abdollahi T, Solhjouy Fard S (Eds) Proceeding of the twentieth Iranian Plant Protection Congress (Shiraz), August 2012. Published by Shiraz University, Shiraz, 197. 